\title{
Serial Magnetic Resonance I maging (MRI) Changes in a Patient with Postoperative Infectious Spondylitis. Correlation with Clinical Features
}

\author{
Jong Min Choi ${ }^{1}$, Ki Joon Kim ${ }^{1 凶}$, Byung Joon Gong ${ }^{1}$, Jee-soo Jang ${ }^{1}$, I-Tae Jang ${ }^{2}$ \\ 'Department of Neurosurgery, Nanoori Hospital Suwon, Republic of Korea \\ ${ }^{2}$ Department of Neurosurgery, Nanoori Hospital Gangnam, Seoul, Republic of Korea
}

\begin{abstract}
We present a case of 58-year-old male patient who underwent post-operative spondylodiscitis. We assessed the correlation between clinical symptoms and blood test results, and serial MRI findings in a patient with postoperative infectious spondylitis who was on treatment. Despite its effectiveness in the diagnosis of infectious spondylitis, MRI findings exhibited slightly delayed correlation with the patient's clinical symptoms and blood test results. Therefore, when treating postoperative infectious spondylitis patients, MR imaging should not be an independent tool, but should be complemented by other examinations and assessment of clinical symptoms.
\end{abstract}

Key Words: Spondylodiscitis; MRI Scan; Complication; Postoperative infection; Spine infection

$\triangle$ Corresponding Author: Ki Joon Kim, Department of Neurosurgery, Nanoori Hospital Suwon, 295, Jungbu-daero, Yeongtong-gu, Suwon-si, Gyeonggi-do, Republic of Korea Tel: +82-2-8065-4235, Fax: +82-2-3445-9755, E-mail: kkj723@hanmail.net

\section{INTRODUCTION}

Patients who undergo surgical treatment for spinal diseases can develop postoperative infectious spondylitis, which is a complication that is difficult to predict and treat. Although surgeons give a high priority to the prevention of postoperative infectious diseases, its prevalence is still reported to be $0.21-3.6 \%$ following spine surgeries ${ }^{1}$. Presently, MRI is used widely in the diagnosis of spondylitis, based on signals that indicate abnormal vertebral bodies or discs as well as changes in the nearby soft tissue. Imaging techniques play a crucial role in the management of the patient's condition as well as in predicting the prognosis. However, only a few studies have reported on serial comparisons of clinical symptoms, blood test results, and MRI findings. In this study, we performed comparative assessment of the factors influencing treatment decision making-including serial changes observed under MRI from the onset of the disease until the end of treatment-in a patient who had undergone spine surgery at our center and developed postoperative infectious spondylitis.

The patient were informed that data concerning the case would be submitted for publication, and they provided consent.

\section{CASE REPORT}

A 58-year-old male patient visited our center with pain in his lower back and right femoral region, as well as radiating pain in his right lower limb. The patient had been suffering from the pain for about 6 months despite of conservative management at a local hospital. From an MRI performed at our center, right paramedian disc rupture at L3-L4 level and degenerative spondylolisthesis grade I and right extraforaminal disc herniation at L5-S1 level were observed. Subsequently, open lumbar microdiscectomy L3-L4 as well as anterior lumbar interbody fusion L5-S1 was planned for the patient. The surgeries were successful and the patient experienced improvement in the radiating pain in his right lower limb, despite having weak pain at the surgery site. Intravenous antibiotics were continued after the surgery until postoperative day (POD) 6. There were no inflammatory symptoms and MRI performed on POD 6 did not show any clear signs of potential inflammation(Fig. 1).

On POD 13, the patient developed pyrexia $\left(38^{\circ} \mathrm{C}\right)$ and lower back pain [numerical rating scale (NRS) 6]. Although the WBC count was normal range, CRP was slightly increased to 2.37. MRI showed fluid collection at L5-S1 disc space, as well as low signal intensity (T1) and high signal intensity (T2) at L5-S1 vertebral endplate (Fig. 2). Furthermore, edema was observed in the bone marrow and surrounding soft tissue, although epidural abscesses were not observed.

We therefore diagnosed the patient as having early-stage postoperative spondylodiscitis and commenced the intravenous administration of empirical antibiotics which is a combination 
of vancomycin and ceftriaxone according to the treatment guideline for vertebral osteomyelitis stipulated by the Infectious Diseases Society of America in 2015 2). For 7 days from the administration of new antibiotics, the patient's fever symptoms alternated between improvement and worsening, while he continued to have persistent lower back pain and an increased CRP of 2.37. From day 8 after commencement of the new antibiotics, fever disappeared. In addition, the patient's lower back pain had improved (NRS 2) and the CRP values had continuously decreased..

After diagnosing the patient with spondylodiscitis, we per-

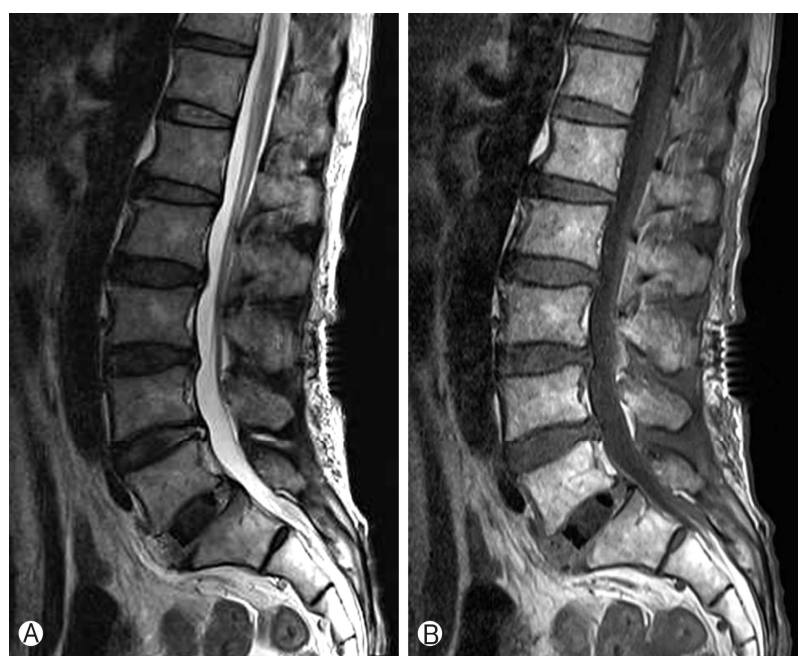

Fig. 1. Postop \#6 T2-weighted image (A), T1-weighted image (B). On MRI, there was no detectable bone marrow edema at the previous ALIF site. ALIF = anterior lumbar interbody fusion.
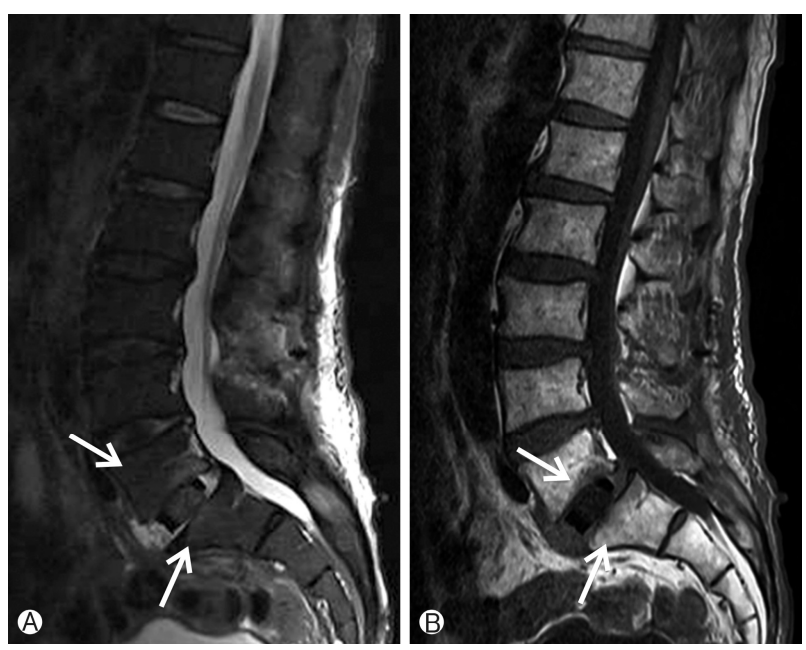

Fig. 2. Postop \#13 T2 fat-suppression image (A), T1-weighted image (B). On MRI, fluid collection in the disc space and bone marrow edema were observed at L5-S1 vertebral body endplate (arrows). formed two follow-up MRI (with a 1- or 2-week interval between sessions) including T2-fat suppression imaging and T1, T2 sagittal, and axial imaging. Despite improvements in clinical symptoms and blood test results, we observed progression of bone marrow edema on MRI (Fig. 3). The patient received intravenous antibiotics for about 4 weeks and was discharged on normalization of CRP and improvements in clinical symptoms. Following his discharge, the patient was instructed to visit our outpatient clinic for assessments of clinical symptoms and blood tests at intervals of 1, 2, 4, and 4 weeks. At each follow-up visit, the patient underwent an MRI examination. From serial MRI

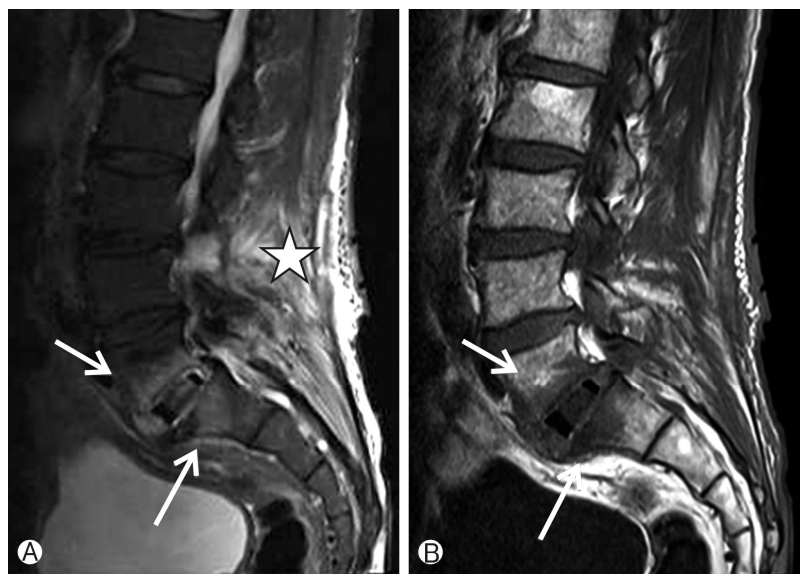

Fig. 3. Postop \#49 T2 fat-suppression image (A), T1-weighted image (B). Despite improved clinical symptoms, bone marrow edema at L5, S1 vertebral body endplate appears to have progressed on MRI (Arrows). Postoperative changes in soft tissue structure are observed near the lesion (star).
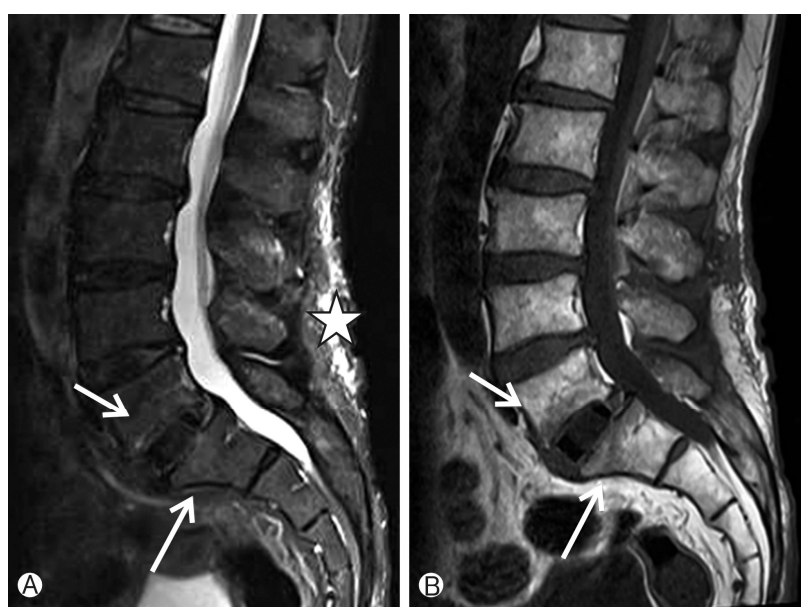

Fig. 4. Postop \#119 T2 fat-suppression image (A), T1-weighted image (B). On MRI, nearly complete loss of bone marrow edema near the lesion is observed, correlating with clinical symptoms (arrows). Soft tissue structures near the lesion returned to normal condition (star). 
NRS

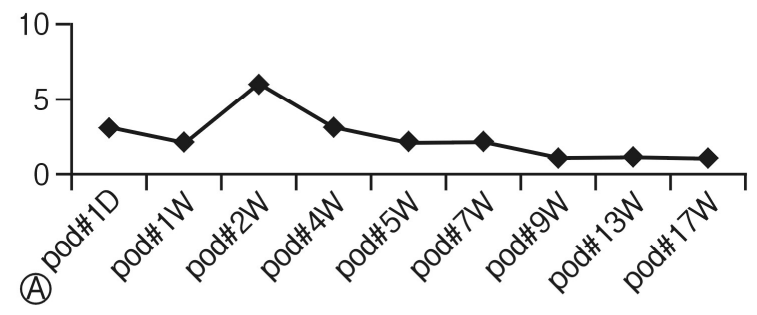

CRP

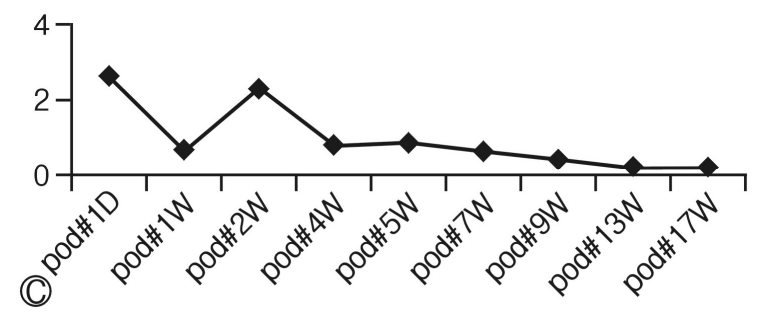

Fever

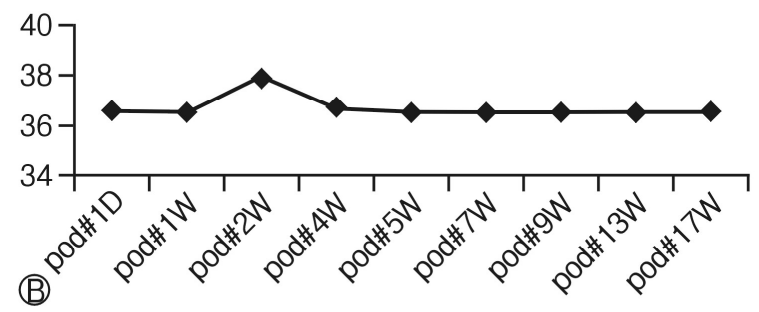

MRI

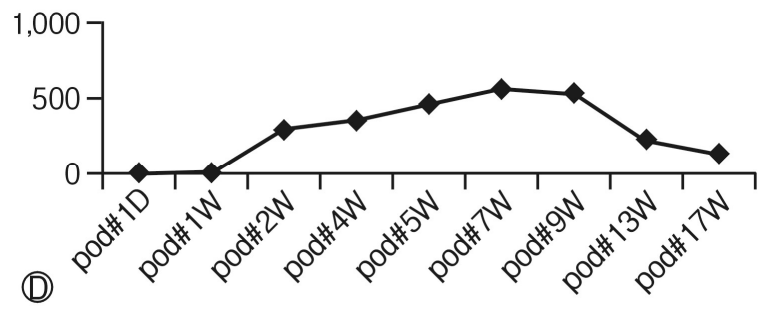

Fig. 5. Comparisons of the degree of pain (A), pyrexia (B), laboratory test (C), and maximal areas of bone marrow edema (D) indicated by signal change under MRI. Unlike clinical symptoms and blood test results, MRI findings do not show correlation. $\mathrm{D}=$ Day, $\mathrm{W}=$ Week.

examinations, we observed a reduction in the extent of bone marrow edema and soft tissue edema in the MR images taken at 3 weeks after discharge. At 11 weeks, we observed remarkable reduction in the extent of these edemas (Fig. 4). In the MR images, changes showing improvement in the soft tissue swellings were first observed, followed by the changes showing improvement in the bone marrow (Fig. 5). We observed a correlation between the clinical symptoms and the laboratory tests in this case report. On the other hand, MRI revealed worsening of edema symptoms, which slowly improved over a relatively longer period of time.

\section{DISCUSSION}

Apart from clinical symptoms, several complementary examination methods have been identified that can help clinicians diagnose spondylitis. Hematological methods include the assessment of $\mathrm{WBC}, \mathrm{CRP}$, and $\mathrm{ESR}^{3)}$. For radiologic diagnosing, MRI is the most frequently used method although alternative options such as single photon emission computed tomography and fluorine-18 fluorodeoxyglucose imaging are also available ${ }^{4)}$. Previous studies have recommended the use of antibiotics for a period of 4-8 weeks for the treatment of spondylitis, because the disease has the clinical characteristic of recurring despite the improvements in clinical symptoms and blood test results ${ }^{5}$. MRI plays a crucial role in the prediction of complementary treatment outcome, and several studies report that MRI findings can assist with accurate treatment outcome and prediction of the time-point for the end of treatment. A previous study has com- pared the changes in MR images of spondylitis patients taken before the treatment and after the final treatment. In this study, we made serial comparisons of the changes in the clinical symptoms, blood test results, and MR images of a single patient, which allowed for the assessment of treatment effectiveness and changes in MRI at different time-points throughout the treatment period.

There are several studies that report on the MRI findings that aid in the diagnosis of infectious spondylitis ${ }^{6,7)}$. These include epidural, disc space, or vertebral body enhancement, or the presence of an abscess or bone marrow edema observed under gadolinium (Gd)-enhanced T1 imaging ${ }^{8}$. A previous study by Gillams et al. had demonstrated that infection was limited to one vertebral body or disc at the early stage of infectious spondylitis, and various signal changes could be observed at T1, T2, or $\mathrm{T} 1-\mathrm{Gd}^{9)}$. Furthermore, during the early stage of recovery after the treatment, contrast for soft tissue was reduced due to $\mathrm{Gd}$ and the extent of bone marrow edema was reduced. Lastly, during the late stage of recovery, high signal could be observed near the lesion in T1-weighted images. In our patient, we observed the signs of late stage recovery after approximately 3 months from treatment completion ${ }^{9)}$. In other words, improvements in MR findings were observed after approximately 2 months after the clinical symptoms and blood test results had returned to normal levels.

Another study suggested that postoperative infectious spondylitis occurs frequently in the posterior parts such as paraspinal muscles and laminectomy sites ${ }^{10}$. Therefore, MRI findings in the posterior parts, such as abscess of paraspinal muscle, are more 
crucial for the diagnosis of postoperative infectious spondylitis, than the findings from lesions of the anterior parts, such as edema of the vertebral body ${ }^{13)}$. Nonetheless, there are limitations in making a diagnosis solely based on radiologic changes observed in the posterior spine, especially in cases where the patient underwent anterior lumbar surgeries, as in the present case, or there is distortion of the MR images caused by the screws used in fusion surgeries.

MRI is a gold standard tool in the diagnosis of infectious spondylitis, especially for confirmation of early stage infection?). However, clinical symptoms and blood test results should be considered in parallel when diagnosing infectious spondylitis. One limitation of MRI-based examination - a paradoxical phenomenon - is that it does not accurately reflect the disease progression $^{12)}$. According to the report by Cheung et al, pain in the infected area occurred in $\geq 90 \%$ of the patients, while pyrexia were observed only in approximately $20 \%$ of the patients. Moreover, ESR and CRP could remain elevated even after the improvements in clinical symptoms. Clinical symptoms and laboratory test results are the important factors that help clinicians take decisions on the different treatment options, such as antibiotic treatment or additional surgeries, whereas MRI findings have limitations as a factor of treatment decision ${ }^{11}$.

There are few limitations to this study. First, serial MRI examinations were performed on a single patient, and hence our findings cannot be applied to all patients. Second, since Gd-enhanced images could not be obtained, it cannot be stated that all radiologic changes were assessed. Nonetheless, our observations of the changes in serial MRI findings in the patient with postoperative infectious spondylitis will help clinicians in deciding on the correct treatment option for similar patients in the future.

\section{CONCLUSION}

Despite its effectiveness in the diagnosis of infectious spondylitis, MRI findings exhibited slightly delayed correlation with the patient's clinical symptoms and blood test results ${ }^{3)}$. In the MR images of these spondylitis patients, bone marrow change, with its paradoxical phenomenon, has a clear limitation as a clinical tool to assess the treatment effectiveness. Therefore, when trea- ting postoperative infectious spondylitis patients, MR imaging should not be an independent tool, but complemented by other examinations and clinical symptoms.

\section{REFERENCES}

1. Gerometta A, Bittan F, Rodriguez Olaverri JC.: Postoperative spondilodiscitis. Int Orthop:433-438, 2012

2. Berbari EF, Kanj SS, Kowalski TJ, Darouiche RO, Widmer AF, Schmitt SK, et al.: 2015 Infectious Diseases Society of America (IDSA) Clinical Practice Guidelines for the Diagnosis and Treatment of Native Vertebral Osteomyelitis in Adults. Clin Infect Dis: 26-46, 2015

3. Kowalski TJ, Layton KF, Berbari EF, Steckelberg JM, Huddleston PM, Wald JT, et al.: Follow-up MR imaging in patients with pyogenic spine infections: lack of correlation with clinical features. AJNR Am J Neuroradiol:693-699, 2007

4. Kouijzer IJE, Scheper H, de Rooy JWJ, Bloem JL, Janssen MJR, van den Hoven $L$, et al.: The diagnostic value of F-FDG-PET/CT and MRI in suspected vertebral osteomyelitis - A prospective study. Eur J Nucl Med Mol Imaging:798-805, 2018

5. Lener S, Hartmann S, Barbagallo GMV, Certo F, Thomé C, Tschugg A.: Management of spinal infection: A review of the literature. Acta Neurochir(Wien):487-496, 2018

6. Sharif HS.: Role of MR imaging in the management of spinal infections. AJR Am J Roentgenol:1333-1345, 1992

7. Yeom JA, Lee IS, Suh HB, Song YS, Song JW.: Magnetic Resonance Imaging Findings of Early Spondylodiscitis: Interpretive Challenges and Atypical Findings. Korean J Radiol:565-580, 2016

8. Gillams AR, Chaddha B, Carter AP.: MR appearances of the temporal evolution and resolution of infectious spondylitis. AJR Am J Roentgenol:903-907, 1996

9. Hanrahan CJ, Shah LM.: MRI of spinal bone marrow: Part 2, T1-weighted imaging-based differential diagnosis. AJR Am J Roentgenol:1309-1321, 2011

10. Ha KY, Kim YH.: Postoperative spondylitis after posterior lumbar interbody fusion using cages. Eur Spine J:419-424, 2004

11. Kim SJ, Lee SH, Chung HW, Lee MH, Shin MJ, Park SW.: Magnetic Resonance Imaging Patterns of Post-Operative Spinal Infection: Relationship between the Clinical Onset of Infection and the Infection Site. J Korean Neurosurg Soc:448-455, 2017

12. Yousem DM, Ihmeidan I, Quencer R, Atlas SW.: Paradoxically decreased signal intensity on postcontrast short-TR MR images. AJNR Am J Neuroradiol:875-80,1991

13. Cheung WY1, Luk KD.: Pyogenic spondylitis. Int Orthop:397404, 2012 